\title{
Rare Presentation of Gallstone lleus with One Stage Laparoscopic Management; the Tragedy, the Comedy and the Solution. Case Report
}

Amr Alnagar ( $\sim$ amr.alnagar@alexmed.edu.eg )

Alexandria University https://orcid.org/0000-0003-3434-6459

Heba Elkoumy

Faculty of Medicine of Alexandria University: Alexandria University Faculty of Medicine

Mohamed Foula

King Fahd Hospital: King Fahad Hospital

Mohamed sakr

Faculty of Medicine of Alexandria University: Alexandria University Faculty of Medicine

Wael Nabil

Alexandria Medicine: Alexandria University Faculty of Medicine

\section{Research Article}

Keywords: pneumobilia, gallstone ileus, intestinal obstruction, single stage laparoscopic repair.

Posted Date: July 7th, 2021

DOI: https://doi.org/10.21203/rs.3.rs-471556/v1

License: (9) This work is licensed under a Creative Commons Attribution 4.0 International License.

Read Full License 


\section{Abstract}

\section{Introduction:}

Gallstone ileus is one of the rare complications of gallbladder stones and is a comparatively unique trigger of alimentary tract obstruction. It involves the development of a cholecystoenteric fistula through which a gallstone has passed into the gastrointestinal tract. Spontaneous resolution of intestinal obstruction in gallstone ileus is extremely rare.

\section{Case presentation:}

We present a case of a 71-year-old female patient presented by right hypochondrial pain for 4 months. Patient gave history of absolute constipation and abdominal distention 2 months before presentation that lasted for 3 days and resolved spontaneously. Imaging showed gallbladder stone and pneumobilia. Laparoscopic exploration showed a cholecysto-duodenal fistula that was divided, opening in the first part duodenum was closed directly in double layers and cholecystectomy was completed successfully.

\section{Conclusion:}

Presence on pneumobilia in patient with gallstones should raise suspicion of fistula between biliary tree and gut. Obstruction component of gallstone ileus can resolve spontaneously in rare occasions. Single stage laparoscopic management of cholecysto-duodenal fistula is safe and feasible in presence of experienced HPB laparoscopic surgeon.

\section{Introduction:}

Gallstone ileus is a comparatively rare trigger of mechanical intestinal obstruction, it occurs in $0.15-1.5 \%$ of cholelithiasis cases and $<0.1 \%$ of ileus cases overall $(1,2)$. The most accepted explanation for such rare clinical entity is migration of stone from gallbladder through a cholecysto-enteric fistula to be impacted distally in the gut (3). Gallstone ileus commonly affects elderly patients with cholelithiasis and can have a high rate of complications(4). Surgical intervention for cases of gallstone ileus involves both stone extraction to relief the obstruction with or without closure of the fistula. Whether surgery should be completed as a one-stage or two-stage operation remains a subject of debate(5). Spontaneous resolution of bowel obstruction caused by impacted stone is extremely rare(6). Fistula can resolve spontaneously but its persistence is a possible aetiology for retrograde cholecystitis or gallbladder cancer.(7-10)

\section{Case Presentation:}

71 years old female patient presented electively to the Hepatobiliary surgery department in Alexandria Main University Hospital with recurrent attacks of right hypochondrial pain and occasional vomiting for four months with history of absolute constipation and abdominal distention two months ago that lasted for 3 days and resolved spontaneously. Patient had a history of hypertension, asthma, and diabetes 
mellitus with no history of previous abdominal surgery.Clinical examination revealed a mild right hypochondrial tenderness and small partially irreducible paraumbilical hernia that patient is known to have for about twenty years.

An abdominal ultrasonography showed a contracted gallbladder over an echogenic shadowing stone. Complementary computerized tomographic scan (CT) showed aerobilia with no intra- or extra- hepatic biliary dilatation and with normal caliber of small and large intestine. Laboratory studies including liver function tests, markers of biliary stasis and inflammatory markers were essentially normal.

A cholecysto-enteric fistula was suspected in view of presence of aerobilia without possible other etiologies like endoscopic retrograde cholangiography and pancreatography (ERCP). The decision was taken to perform a diagnostic laparoscopy by one of our experienced HPB laparoscopic consultants using the conventional four trocar approach for laparoscopic cholecystectomy(11).

Exploration revealed a fistula between body of gallbladder and first part duodenum (Fig. 1), fistula was disrupted (Fig. 2) and opening in duodenum was closed in double layers. (Fig. 3). That was followed by Uneventful laparoscopic cholecystectomy and through irrigation of the abdomen then insertion of $18 \mathrm{~F}$ drain in Morison's pouch. At the end of the procedure, the paraumblical hernia was reduced, defect was closed and onlay prolene mesh was fixed to anterior rectus sheath. The patient had a smooth postoperative course and was discharged on the 5th post-operative day.

\section{Discussion:}

Biliary tree can spontaneously form different types of fistulae as bilio-enteric, bilio-bronchopleural and bilio-vascular. Bilio-enteric fistula is a rare complication of cholelithiasis with an estimated incidence of less than 5\%.(12) Specifically, cholecysto-duodenal fistula (CDF), first described by Corvousier in 1980, represents the most common type (80\%) of bilio-enteric fistula which also includes cholecysto-colonic, cholecysto-gastric, cholecysto-jejunal, and choledocho-duodenal fistulae, in order. CDF usually affects elderly females with other comorbidities.(13)

The pathogenesis of CDF represents two main theories: 1) repeated attacks of cholecystitis leading to adhesions between the gallbladder and the duodenum with consequent erosion and fistulization, and 2) pressure necrosis of gallbladder by a large gallstone. Other causes of CDF include peptic ulcer, trauma, malignancy and inflammatory bowel disorders.

After development of CDF, gallstones can freely gain access to the gastrointestinal tract and CDF has a spectrum of clinical presentations based on the gallstones size. The classical full-blown picture is gallstone ileus (GSI) with complete intestinal obstruction.(14) CDF may be asymptomatic if the gallstones size is less than $25 \mathrm{~mm}$ with unobserved passage. It may also be an incidental finding during an elective cholecystectomy, or cause recurrent attacks of ascending cholangitis.(14) Gallbladder cancer can complicate the CDF and is found in $0.82 \%$ of patients with CDF.(15) Rarely, GSI may present with 
recurrent attacks of partial intestinal obstruction indicating movement of gallstones. Some patients may complain of vague non-specific symptoms as abdominal pain, diarrhea and stetaorrhea.

Indeed, diagnosis of CDF is challenging owing to absence of any specific symptoms, signs, laboratory markers or radiological findings. Preoperative diagnosis is achieved in only $17 \%$ of patients, however, it is crucial to avoid unplanned procedures and undesirable preventable complications. Rigler's triad, first described in 1941, is pathognomonic for GSI and consists of intestinal obstruction, pneumobilia (areobilia), and ectopic gallstones.(16) Intestinal obstruction is reported in $70 \%$ of patients with bilioenteric fistula.(17)

Pneumobilia, the most common radiological finding associated with CDF, is detected in one third of these patients. Radiologically, the presence of pneumobilia is always abnormal and, hence, may provide the first clue of the bilio-enteric fistula. However, it may be misinterpreted to other pathologies as emphysematous gallbladder, hepatic abscess or portal vein gas. It can also be seen in patients with sphincter of Oddi dysfunction or after biliary tree intervention as ERCP. $(13,18)$ The trans-abdominal ultrasonography is not accurate in detecting CDF, however, it could raise the suspicion for bilio-enteric fistula and indicate further imaging as in case of a contracted gallbladder with absence of previouslynoted gallstones.(12) Magnetic resonance cholangiopancreatography (MRCP) delivers further data about biliary tree anatomy but is only indicated in certain situations.

There are debates on the best management approach for CDF, but it mainly depends on its clinical presentation. For $\mathrm{GSI}$, the most common presentation, surgical management is standard without a consensus on the best approach: one-staged versus two-staged approach.(16) Furthermore, some authors have reported a successful conservative trial with spontaneous resolution of GSI.(17, 19, 20) While incidentally-discovered CDF is feasibly managed as one-staged procedure including cholecystectomy and fistulectomy or fistula repair. Laparoscopic management can be safely performed with experienced hands and should be performed in a well-equipped specialized center.(5) However, a high rate of conversion to laparotomy is expected and has been frequently reported due to dense adhesions, bleeding, multiple fistulae. $(12,13)$

There are multiple interesting points in our case. First point is her clinical presentation. She was an elderly lady with multiple comorbidities and presented with recurrent typical biliary colic. A brief obstructed paraumbilical hernia was blamed for her transient attack of abdominal distention and constipation which resolved spontaneously after three days without medical advice. However, her ultrasonography showed a contracted gallbladder and her CT abdomen, requested by the radiologist, revealed pneumobilia. She had no other abnormal or suspicious laboratory or imaging findings. At this point, an incidental finding of CDF was assumed, though, the complete history and workup were not enough to definitely establish a preoperative diagnosis of CDF. Second interesting point is the management approach. Based on the diagnostic laparoscopy, a clear fistula was found to connect the gallbladder and first part of duodenum. We opted to perform a laparoscopic one-stage procedure including cholecystectomy and fistula repair which was successfully completed and the patient had a smooth uneventful postoperative course. We 
preferred the laparoscopic approach, based on our experience, to avoid the complications of laparotomy and its lengthy postoperative recovery. Third interesting point is the spontaneous resolution of her gallstone ileus without diagnosis or intervention. Retrospectively and based on pneumobilia and presence of CDF, her transient intestinal obstruction was better attributed to CDF rather than obstructed paraumbilcal hernia. The spontaneous resolution of GSI has been only reported in few articles after a trial of conservative approach for otherwise-unfit patients.(17)

\section{Conclusion:}

Cholecysto-enteric fistula is rare complication of cholelithiasis and the presence of pneumobilia in patient with gallstones should raise suspicion of fistula between biliary tree and gut. High index of suspicion, based on detailed history and imaging findings, is important for its preoperative diagnosis. Obstruction component of gallstone ileus can resolve spontaneously in rare occasions. Single stage laparoscopic management is feasible and safe approach when performed by experienced HPB laparoscopic surgeon. Despite its rarity, gallstone ileus can be unnoticedly resolved.

\section{Declarations:}

i. Funding (information that explains whether and by whom the research was supported): This case report did not receive any specific grant from funding agencies in the public, commercial, or not-for-profit sectors.

ii. Conflicts of interest/Competing interests (include appropriate disclosures): The authors declare that they have no conflict of interest.

iii. Ethics approval (include appropriate approvals or waivers): Not applicable.

iv. Consent to participate (include appropriate statements: Not applicable.

v. Consent for publication (include appropriate statements): Consent is available upon request

vi. Availability of data and material (data transparency): Data is available upon request.

vii. Code availability (software application or custom code): Not applicable.

viii. Authors' contributions

Alnagar.A

Manuscript writing- operating surgeon.

Heba Elkoumy

Reviewing literature 
Mohamed S. Foula

Reviewing literature.

Mohamed A. Sakr

Manuscript editing.

Wael Nabil

Operating consultant - final drafting.

Patient Perspective:

I had recurrent right hypochondrial pain, doctors went carefully through my history and my imaging result and shared with me that I could have a rare complication of gallstone and that I was lucky that the intestinal obstruction component resolved spontaneously. After surgery I felt much better, and I was grateful that my surgery could be done laparoscopically.

\section{References:}

1. Halabi WJ, Kang CY, Ketana N, Lafaro KJ, Nguyen VQ, Stamos MJ, et al. Surgery for gallstone ileus: a nationwide comparison of trends and outcomes. Annals of surgery. 2014;259(2):329-35.

2. Nakao A, Okamoto Y, Sunami M, Fujita T, Tsuji T. The oldest patient with gallstone ileus: report of a case and review of 176 cases in Japan. The Kurume medical journal. 2008;55(1+2):29-33.

3. Beuran M, Ivanov I, Venter M. Gallstone ileus-clinical and therapeutic aspects. Journal of medicine and life. 2010;3(4):365.

4. Shioi Y, Kawamura S, Kanno K, Nishinari Y, Ikeda K, Noro A, et al. A case of gallstone ileus displaying spontaneous closure of cholecystoduodenal fistula after enterolithotomy. International journal of surgery case reports. 2012;3(1):12-5.

5. Inukai K. Gallstone ileus: a review. BMJ open gastroenterology. 2019;6(1):e000344.

6. Farooq A, Memon B, Memon MA. Resolution of gallstone ileus with spontaneous evacuation of gallstone. Emergency radiology. 2007;14(6):421-3.

7. Clavien PA, Richon J, Burgan S, Rohner A. Gallstone ileus. British Journal of Surgery. 1990;77(7):73742.

8. Wakefield E. Cholecystoenteric fistulas. Surgery. 1963;54:716. 
9. Cooperman AM, Dickson ER, ReMine WH. Changing concepts in the surgical treatment of gallstone ileus: a review of 15 cases with emphasis on diagnosis and treatment. Annals of surgery. 1968;167(3):377.

10. Bossart PA, Patterson AH, Zintel HA. Carcinoma of the gallbladder: a report of seventy-six cases. The American Journal of Surgery. 1962;103(3):366-9.

11. Gurusamy KS, Vaughan J, Rossi M, Davidson BR. Fewer-than-four ports versus four ports for laparoscopic cholecystectomy. Cochrane Database of Systematic Reviews. 2014(2).

12 Meena R, Srivastava VK, Roy S, Khanna R. Incidental cholecystojejunal fistula: a rare complication of gall stone disease. MOJ Surg [Internet]. 2020 Dec 17;8(4):91-2. Available from: https://medcraveonline.com/MOJS/incidental-cholecystojejunal-fistula-a-rare-complication-of-gall-stonedisease.html

13 Jung H II, Ahn T, Cho SW, Bae SH, Lee MS, Kim CH. Incidental cholecystojejunal fistula treated with successful laparoscopic management. 2014;276-8.

14 Arslan B, Yilmaz T, Erol V, Gulay H. AAsymptomatic cholecysto-enteric fistula and gallstone ileus: Two case reports. Ann Med Res. 2018;26(0):1.

15 Ravikumar R, Williams JG. The operative management of gallstone ileus. 2010;279-81.

16 Mirza Gari MK, Eldamati A, Foula MS, Al-Mulhim A, Abdulmomen AA. Laparoscopic management for gallstone ileus, case report. Int J Surg Case Rep [Internet]. 2018;51:268-71. Available from: http://www.ncbi.nlm.nih.gov/pubmed/30227374

17 De Giorgi A, Caranti A, Moro F, Parisi C, Molino C, Fabbian F, et al. Spontaneous resolution of gallstone ileus with giant stone: A case report and literature review. J Am Geriatr Soc. 2015;63(9):1964-5.

18 Yeniocak S, Az A, Canıpek E, Ekinci Biçkici B, Metiner M, Demirel A. A rare case: Asymptomatic spontaneous pneumobilia. J Surg Med. 2019;3(12):12-4.

19 Bajracharya A, Pathania O, Adhikary S, Agrawal C. A case of colonic gallstone ileus with a spontaneous evacuation. Heal Renaiss [Internet]. 2011 Jan 1;9(1):47-9. Available from: https://www.nepjol.info/index.php/HREN/article/view/4363

20 Farooq A, Memon B, Memon MA. Resolution of gallstone ileus with spontaneous evacuation of gallstone. Emerg Radiol. 2007;14(6):421-3.

\section{Figures}




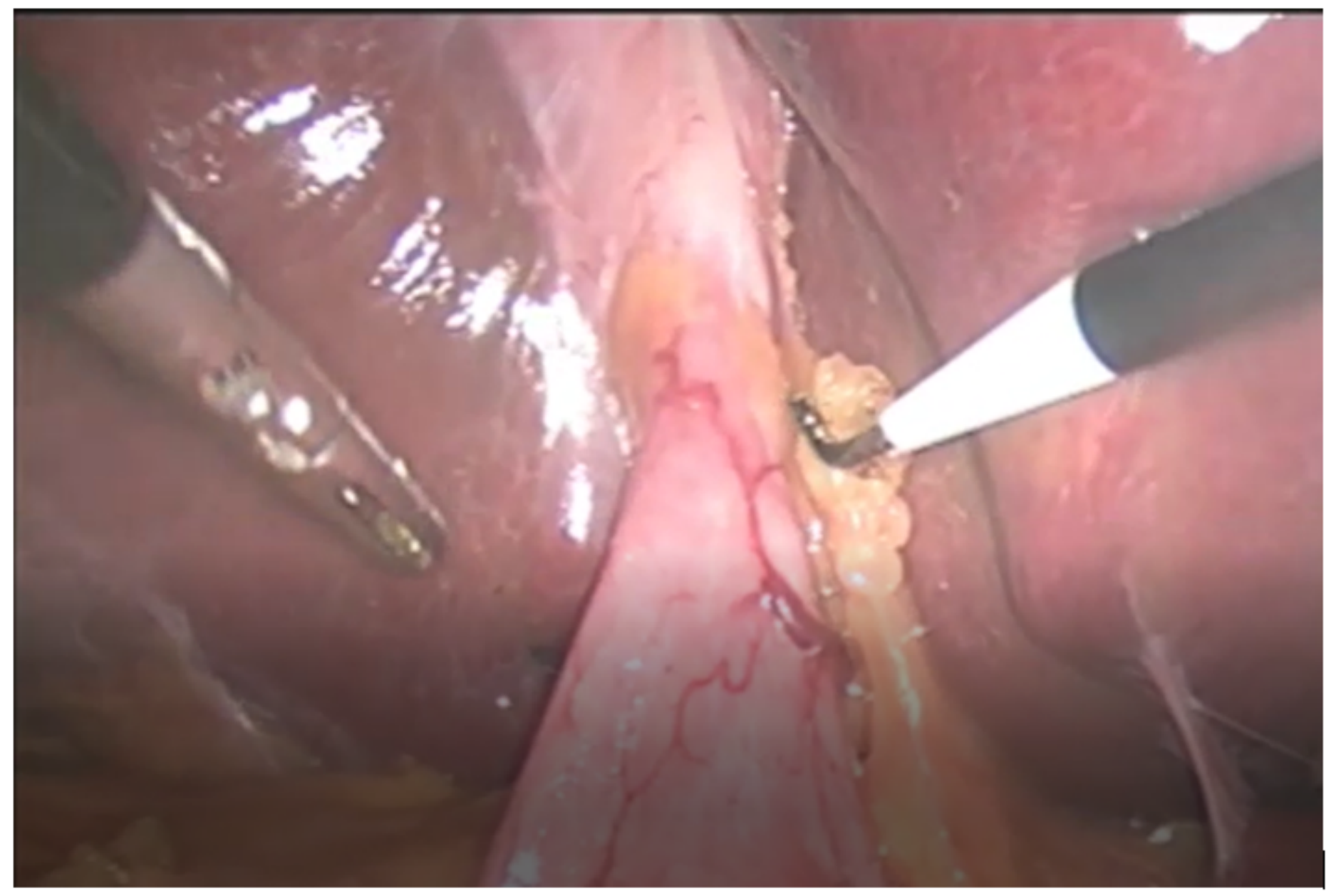

Figure 1

Fistula between body of gallbladder and first part duodenum. 


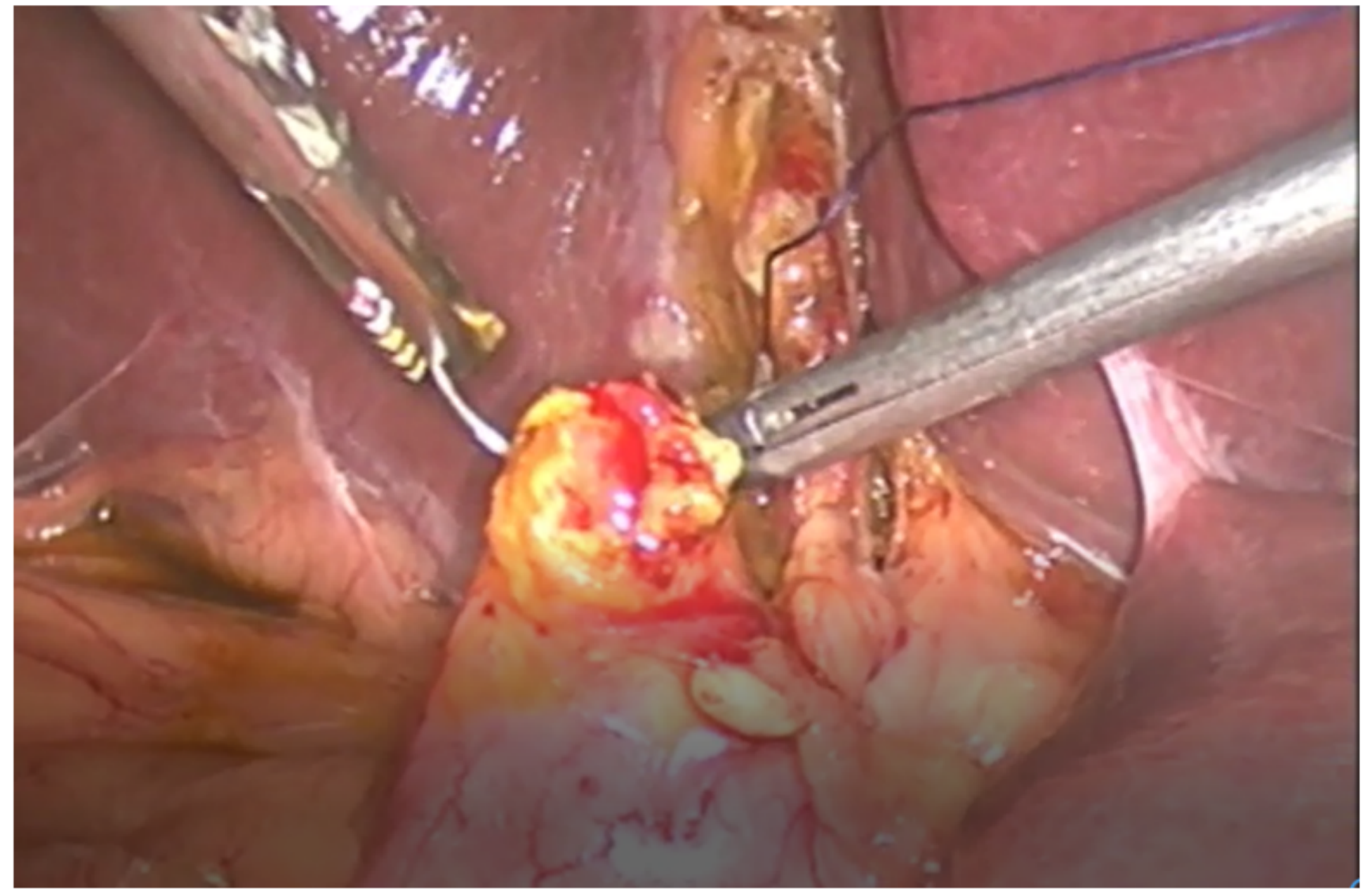

Figure 2

Disruption of fistula between gallbladder and first part of duodenum and start of repair. 


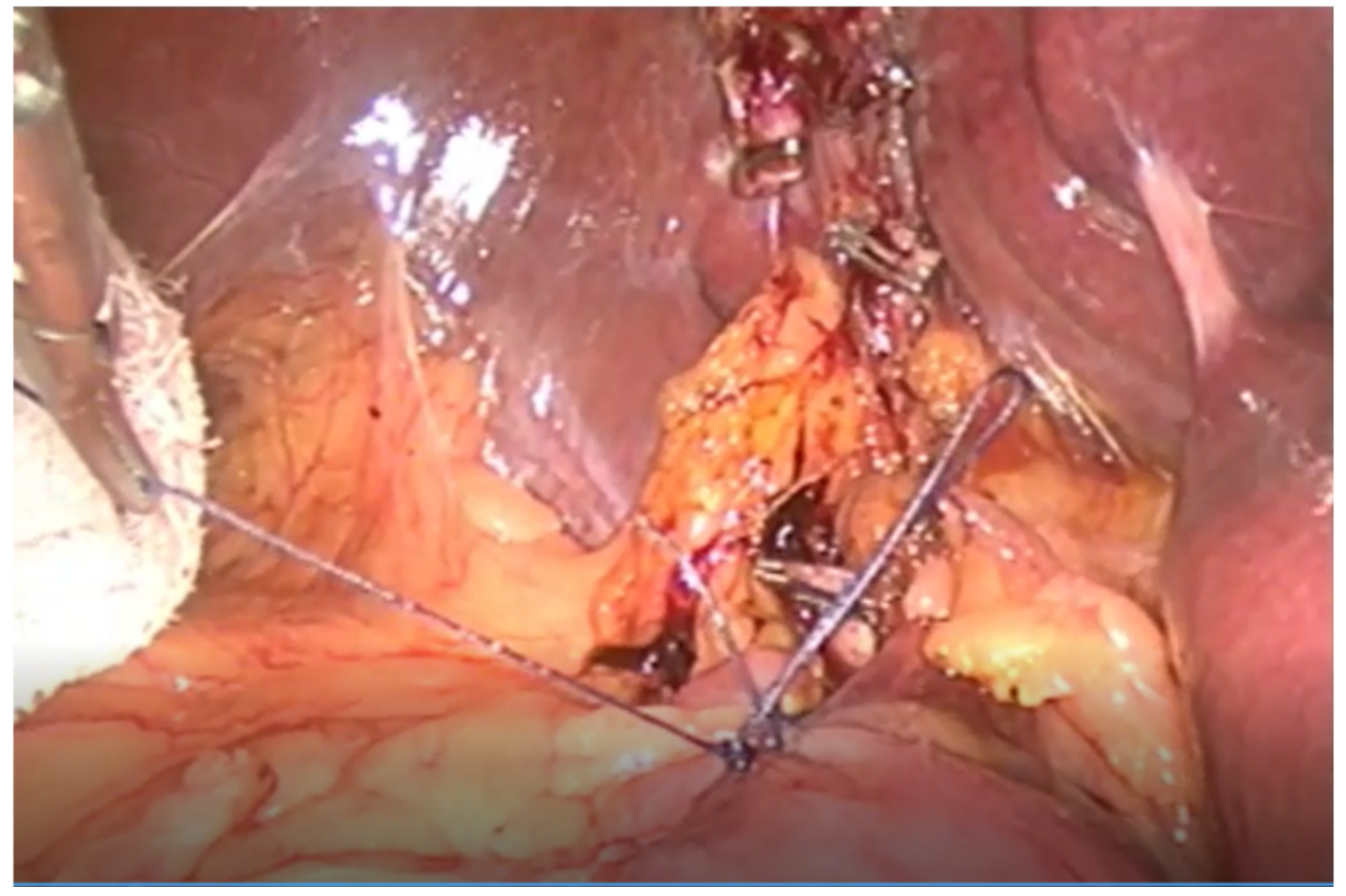

Figure 3

Closure of duodenal opening in double layers.

\section{Supplementary Files}

This is a list of supplementary files associated with this preprint. Click to download.

- AREChecklistofinformationtoincludewhenwritingacasereport.docx 